\title{
Flaporhexis: Alternative Way to Make a Femtosecond Laser Corneal Flap*
}

\author{
Wolfgang A. Pfaeffl \\ The Augen Versorgungs Zentrum, Weilheim, Germany \\ Email: pfaeffl@msn.com
}

Received January 7, 2013; revised February 10, 2013; accepted February 18, 2013

\begin{abstract}
Flaporhexis, a technique to make the femtosecond laser corneal flap was developed primarily to reduce the mechanical trauma associated with separating the corneal tissue by taking advantage of the cornea's natural anatomy and was first described in a peer reviewed artice in 2008. The anterior part of the cornea tends to be stronger, thereby allowing tearing (Greek "rhexis") along the preformed cuts of the femtosecond photo disruption. Flaporhexis differs from Binder's technique of "hinge opening" as published in 2006 by opening the flap from the opposite side. If a femtosecond flap lift is performed correctly, the surgeon will find a virgin, uniform, dry stromal bed, which had not been manipulated by any surgical instrument and is therefore perfect for wavefront guided laser surgery as "Sub-Bowman's Keratomileusis".
\end{abstract}

Keywords: Flaporhexis; Hinge Opening; Sub-Bowman’s Keratomileusis; Flap Lifter; Flaporhexis Forceps

\section{Introduction}

The first description of "flaporhexis" was published in 2008 [1]. A report that also used this term flaporhexis for a variation on a LASIK flap relift technique described in 2000 by Jean-Luc Febbraro [2], was published in 2012 [3]. This present article describes the initial concept of flaporhexis, the origin of its name and its advantages and limitations compared with traditional forms of generating the femtosecond laser flap. Two special instruments, the
Femto-flapLIFTER \& Flaporhexis forceps, have been developed to safely perform the technique (Figure 1).

It has to be pointed out that much of what is written in this article is condensed, collective experience and discussion by a whole surgical community, which had to implement the fascinating tool of femtosecond laser technique to make femto LASIK a safe procedure.

The surgical approach of Flaporhexis as a special type of femtosecond laser flap making is derived from what is

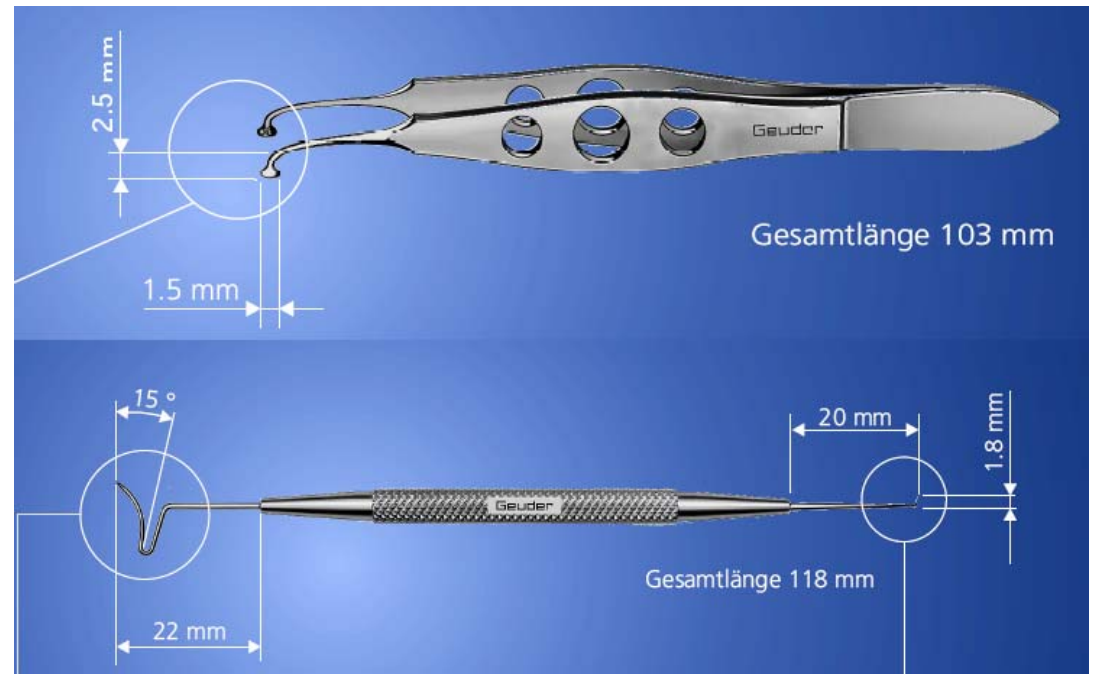

Figure 1. FemtoflapLIFTER\& Flaporhexis forceps, specials instruments developed to safely perform the technique.

\footnotetext{
"No financial interest in the materials or methods presented. The author does not earn royalties for the development or promotion of the mentioned instruments.
} 
commonly known as the can-opener technique [2]. It is used when a microkeratome flap has to be relifted for retreatment and enhancement procedures using the excimer laser. Febbraro does not use this expression in his article, but to the best of my knowledge it is the first description of this technique. It has become a colloquial term among refractive surgeons because it provides the idea of reopening a flap in a way similar to that of opening a can. Choi and Wilson presented a video with a similar technique for Keratom-LASIK at the annual meeting of the American Academy of Ophthalmology, Dallas, Texas, USA in October 2000 as "LASIK Enhancement: How to lift the flap to zap in a snap". In his article of 2010 [3] Wilson adopted the terminus "Flaporhexis" for any technique of lifting flaps, no matter of microkeratom or femtosecond laser origin, even the technique of "Flaporhexis" was primarily developed as alternative to safely create a primary flap of the femtosecond laser in virgin corneal tissue which had been never laminated before.

\section{Etymology of Flaporhexis (Scheme 1)}

The new technology of femtosecond lasers became commercially available in 2001. The first video presentations of flaporhexis in 2004 were shown in workshops that taught and propagated this bladeless flap making procedure. Flaporhexis was developed to reduce the mechanical trauma of separating the corneal tissue along the preformed spaces of photodisruption and to find a way of simulating the easy lifting of a previous microkeratome flap. The anterior part of the cornea, especially Bowman's membrane is a very solid structure, thereby allowing tearing (greek: "rhexis") with an appropriate instrument. This explains the English-Greek composition of the term flap + rhexis.



Scheme 1. Principle of “Flaporhexis”.
A refractive surgeon may think of the postLASIK eye as a simple can lid to be opened while the uninitiated patient wonders why his/her eye is being abused during a flap lift procedure. In a situation where a fully conscious and potentially anxious patient can listen to the conversation between the surgeon and his staff, it is advisable to use professional terminology to avoid stress to the patient. This is the advantage of the unbiased public use of a medical term of Latin or Greek descent, which also proudly honors the history of our western medical tradition. We need more of these unbiased professional terms or at least use positive colloquial expressions such as "bladeless LASIK" for our patients.

Where flaporhexis differs from Perry Binder's conventional technique of "hinge opening" [4] is by opening the flap from the opposite side of the hinge of the femtosecond laser flap (see scheme: principle of Flaporhexis).

If a femtosecond flap lift is performed correctly by flaporhexis, the surgeon will find a virgin, uniform, dry stromal bed, which has not been manipulated by any surgical instrument and is therefore perfect for wavefront guided laser surgery. Initially, we introduced the term flaporhexis to be used exclusively for making the flap using femtosecond laser technology. Here no pre-existing cut is used but a virtual, pre-determined, breaking point between the potential flap and the stroma. Having understood the etymological migration of words, the pictorial expression of flaporhexis may now of course be transferred to similar ways of making the corneal flap.

\section{Step-by-Step Guide to Flaporhexis}

In the following, the step-by-step description is a condensed summary of a presentation given at the ASCRS meeting in San Diego in 2007.

The photo disruption parameters applied to the cornea are $1.0 \mathrm{~mJ}$ bed energy and $1.2 \mathrm{~mJ}$ side cut energy with spot separation of $8 \mu \mathrm{m}$ and a line separation of $8 \mu \mathrm{m}$, a superior hinge and a $90^{\circ}$ side cut angle. The flap diameter is preset at a maximum of $9.3 \mathrm{~mm}$ but may be changed according to the individual anatomy of the cornea.

1) Enter the side cut with the short hook side of the FemtoflapLIFTER (Geuder AG Heidelberg) or the SeibelIntraLASIK Flap Lifter (Rhein Medical Inc., Tampa, Florida) at the 9 o'clock or 3 o'clock position by pushing down vertically into the gap till you "pop" into the gutter (Figure 2).

2) Turn the tip by 90 degree to open the gutter and make an $180^{\circ}$ semicircular swing to the contra-lateral side following the tip inside the edge of the gutter (Figures 3 and 4 ).

3) Use the specially designed flaporhexis forceps (Geuder AG, Heidelberg, Germany) to slip the head of the forceps completely into the interface space at the 6 


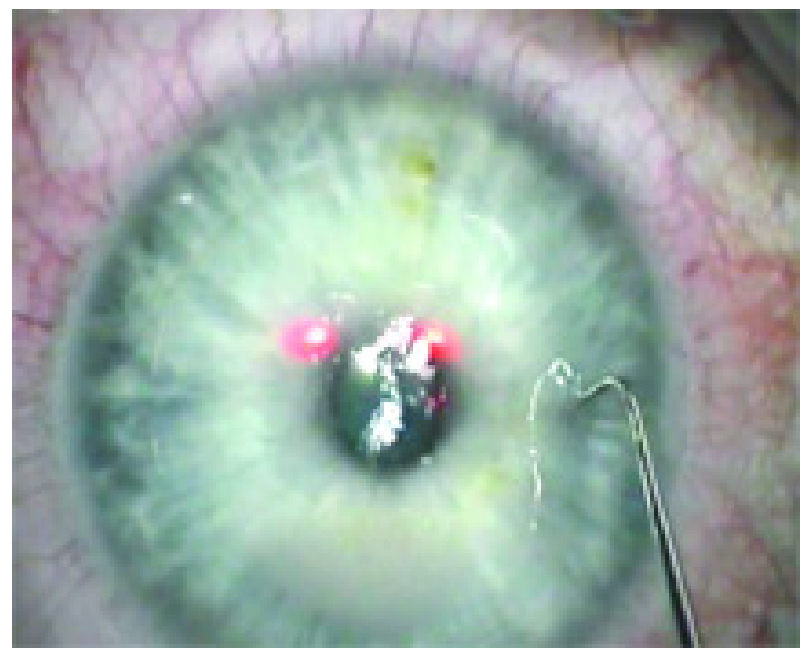

Figure 2. Entering the side cut of the corneal flap.

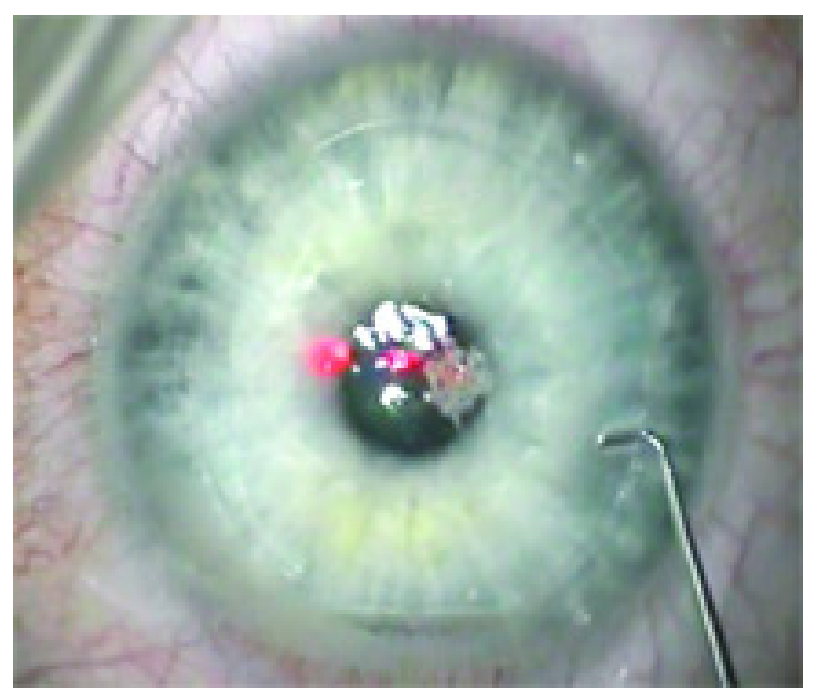

Figure 3. Turning the tip by 90 degree.

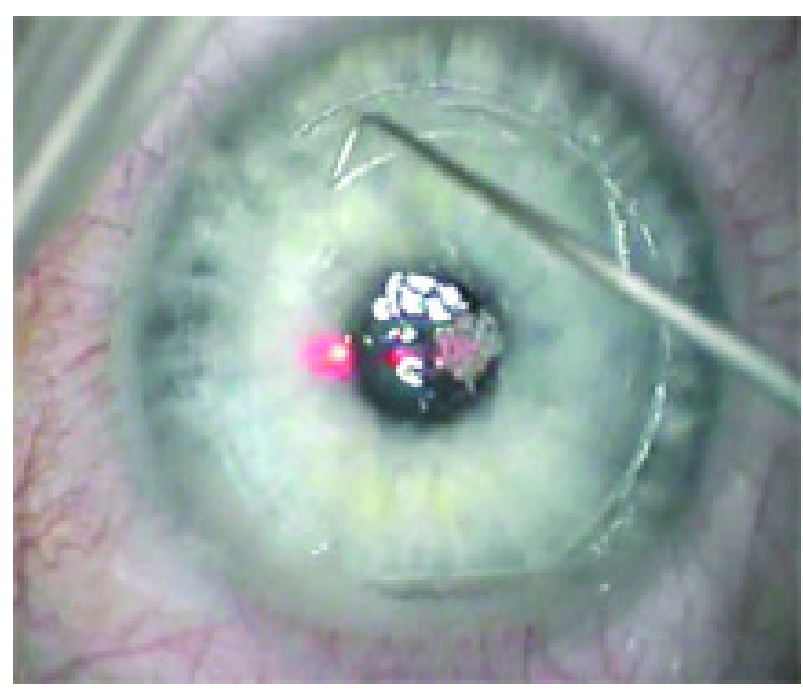

Figure $4.180^{\circ}$ semicircular swing. o'clock position. Firmly grab the flap (Figure 5).

4) Lift the flap with a strong and determined pull back to the hinge and lay it down (Figure 6). Beginners are advised to do the full $180^{\circ}$ swing of step 2 because from this point onwards, the tension built up by the flaporhexis forceps runs toward the edges of the hinge, comparable to the manipulations necessary to perform a capsularhexis [5]. Having gathered more experience and confidence, the surgeon tends to reduce the opening of the side-cut step by step. But it must be understood that, in the case of an incomplete side cut, the tension build-up in the gutter can cause a deviation of the "rhexis" away from the gutter causing a "bite out" (Figure 7), an "partial flap" (Figure 8) or a "hemiflap" (Figure 9).

Once the flap is folded back, the surgeon should find a virgin, uniform, dry stromal bed that is ready for wavefront-guided treatment. When the flap is refloated, settled

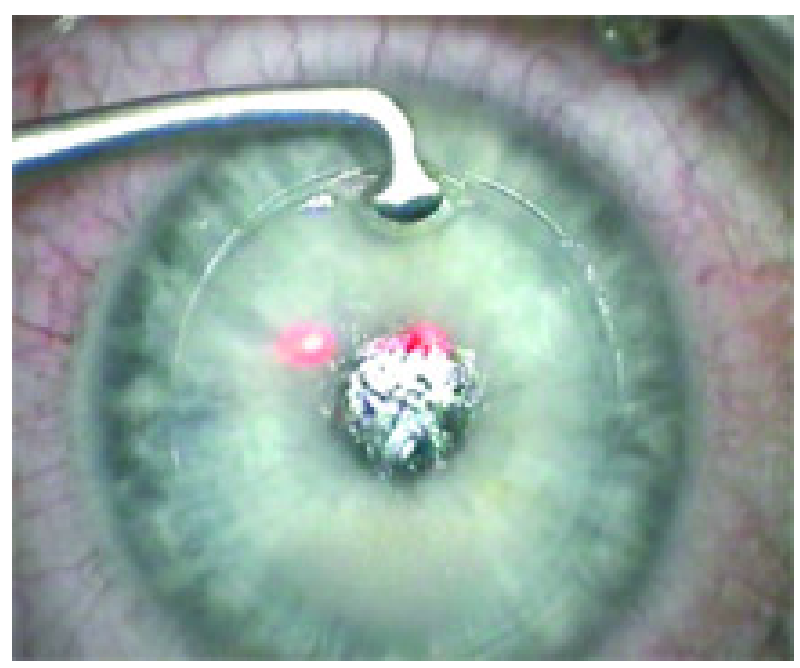

Figure 5. Firmly grab the corneal flap with the flaporhexis forceps.

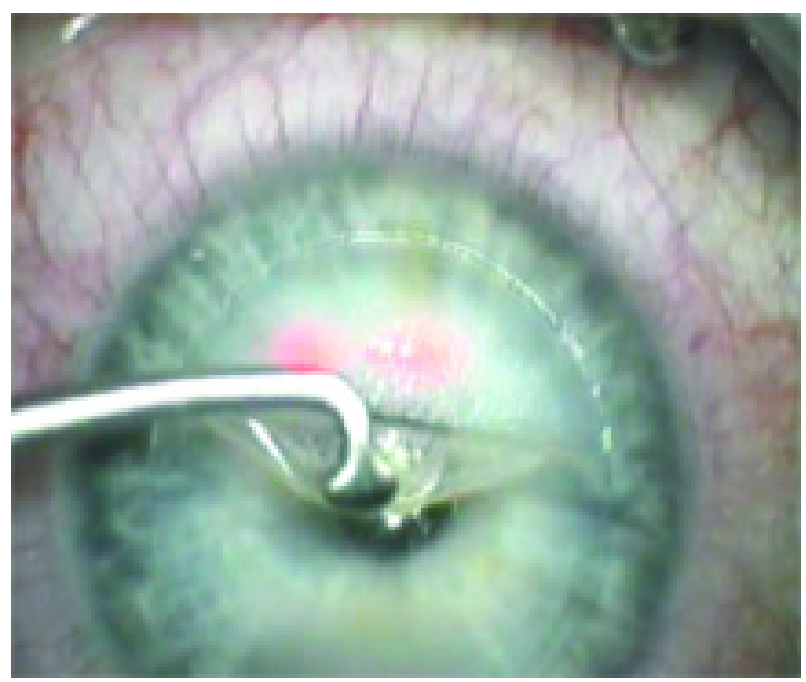

Figure 6. Pull back to the hinge. 


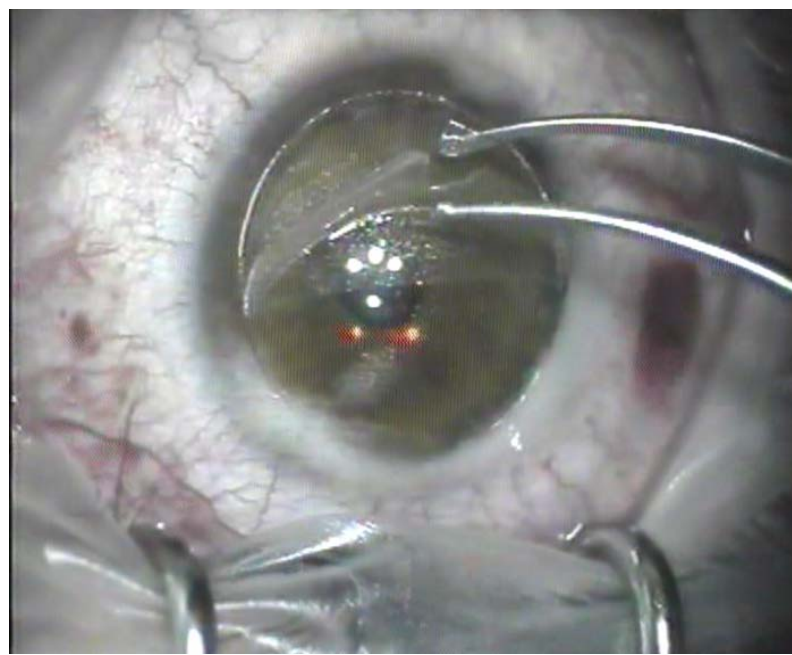

Figure 7. Corneal flap bite out.

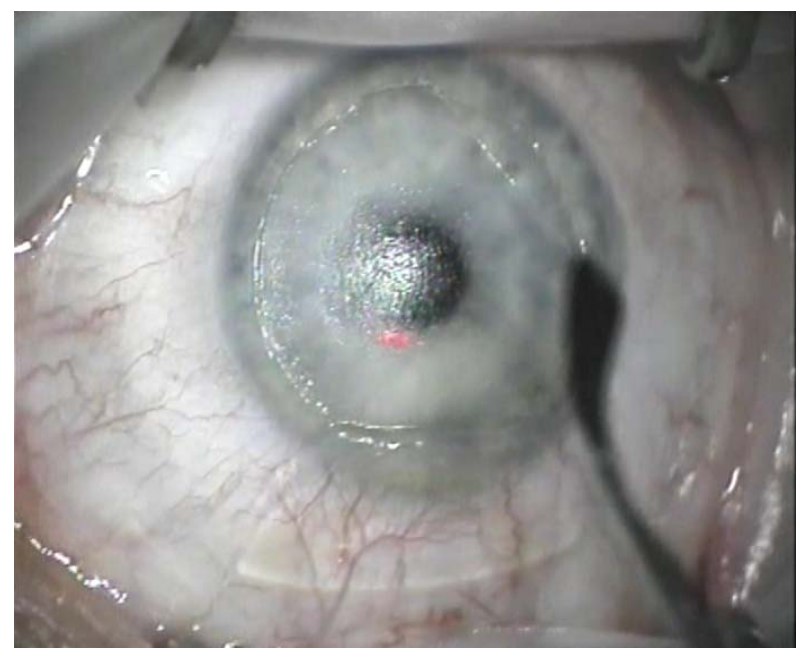

Figure 8. Partial corneal flap.

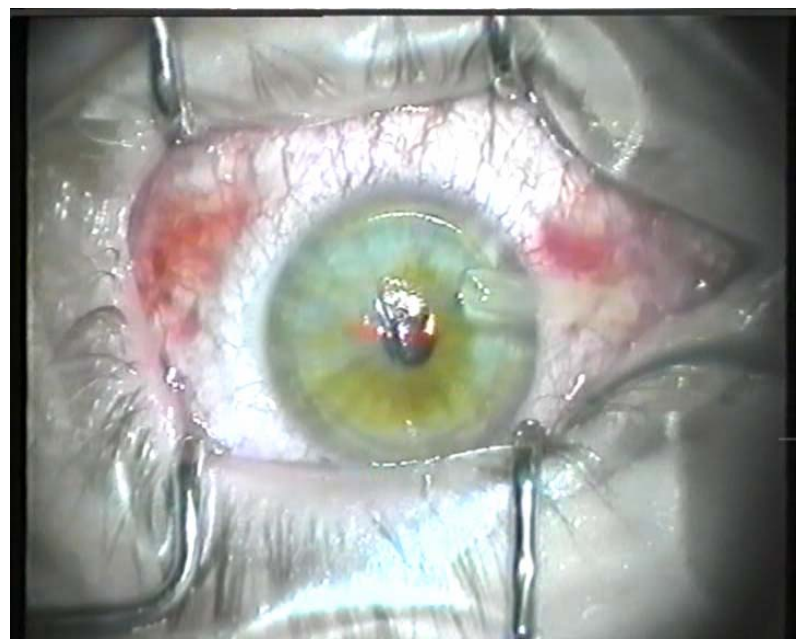

Figure 9. Hemi flap.

and adapted with a wet Methocel sponge after the laser ablation, a slight imprinted mark of the forceps is stainable with fluorescein for some hours, but the flap is perfectly adapted in its gutter. In our experience, the uniform design of flaps created by this femtosecond laser technique transfers the optical higher-order aberrations on the surface down to the lower layer of the ablation area of the corneal stroma better than in any keratome technique. Plus, the $90^{\circ}$ degree side cut geometry is less prone to epithelial invasion than the oblique edge design of keratom flaps.

\section{Discussion}

Perry Binder's hinge-opener technique [4] is a classic and safe technique for accessing the stromal bed and to creating the flap by entering the corneal tissue near the hinge (Figure 10). The steel instrument, a FemtoflapLIFTER or a Seibel-IntraLASIK Flap Lifter, enters the side cut, and the flap is stretched (Figure 11) against the counter resistance of the peripheral unseparated part of

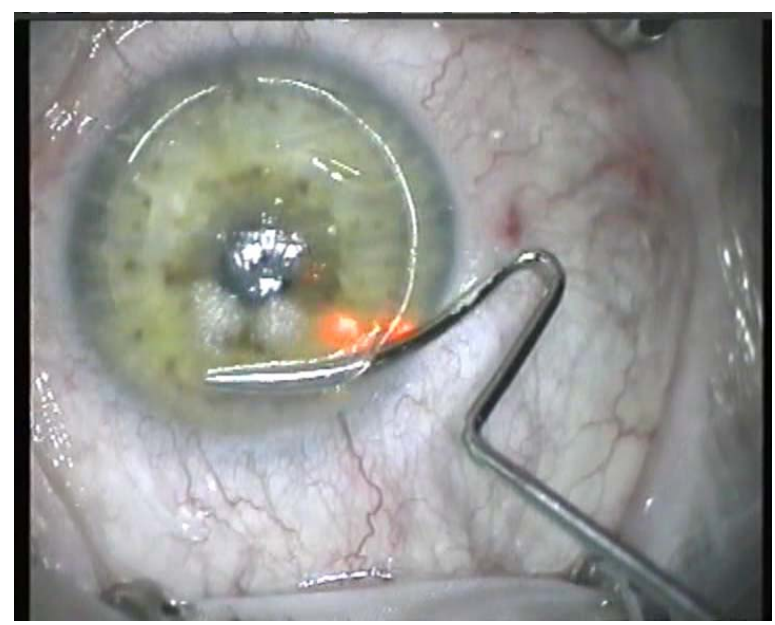

Figure 10. Entering the corneal tissue near the hinge.

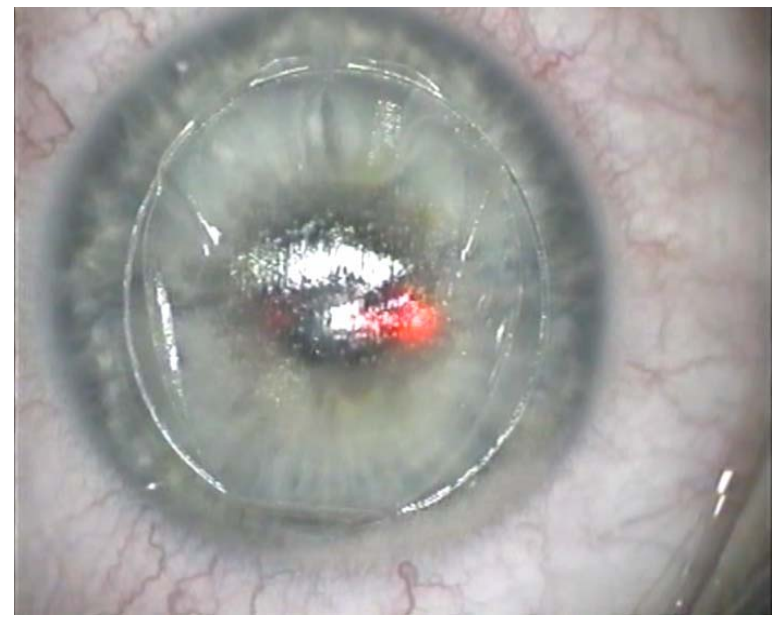

Figure 11. Corneal flap stretched after dissection. 
the flap, or moved in a "windshield-wiper" motion across the cornea [4]. Surgeons who have started on 5, 10, or 15 Hz-femtosecond technology have chosen this technique because of its easy adaptability under the circumstances of rather imperfect photo disruption at the beginning of the aera of femtosecond laser corneal surgery. One disadvantage, however, is that the steel instrument intrudes into a preformed corneal space, and there is a potential for via falsa penetration (Figure 12) of the tissue with the steel instrument in the case of imperfect photo disruption of the corneal layers. Due to the varying initial interactions between the corneal tissue and the $\mathrm{CO}$ bubbles formed by the femtosecond laser, effects of vertical or horizontal breakthrough may occur. The forming of an opaque bubble layer of softened and weakened tissue enables the perforation by the rectangular design of the Seibel-IntraLASIK Flap Lifter. To avoid this, the FemtoflapLIFTER has been given a curved physiologically better design (Figure 1). In some cases, the surgeon may not be able to exit the side cut at the opposite side of the hinge. To solve this problem the surgeon has to find variations from the intended plan. He can now widen the gap between corneal stroma and flap from hinge to periphery by moving the instrument in a "multilane" fashion several times across the cornea to widen the dissection space. Generally, in the hinge opening technique, the cooperation of the patient is needed [4] to counteract the said manipulations of the instruments by activation of the patient's eye muscles (Figure 12). Some patients experience frightening sensations here because they expect a gentle "bladeless" LASIK. In a complicated case, the intrusion of the instrument may also increase the chance of tissue damage and potential implantation of epithelial cells or detritus into the interface due to prolonged manipulation. Thus, in otherwise uneventful treatments, microstriae are sometimes visible in the first postoperative week due to the mechanical stress on the flap's inner

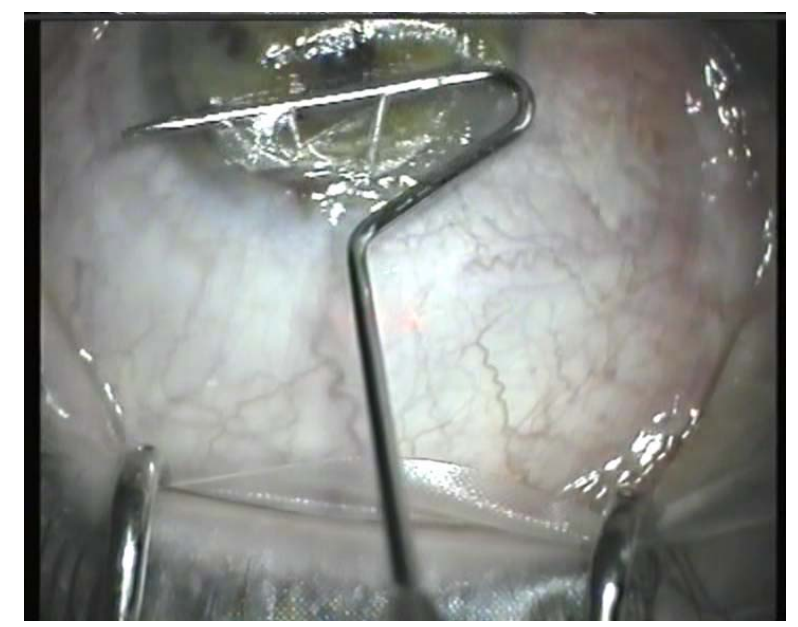

Figure 12. Via falsa penetration of the corneal flap. side (Figure 11). Wavefront-guided treatments are preferably performed on an untouched stromal bed with uniform dryness as produced by flaporhexis. They may suffer from the mechanical stress of the hinge opening technique due to its prolonged manipulation in the corneal interface. Plus, the prolonged manipulation due to incomplete photodisruption of the side cut or the interface may open the gate to the very rare cases of epithelial ingrowths in femtosecond laser flap creation.

We completely converted our flap lifting to flaporhexis in February 2005. To date, we have created more than 5000 consecutive flaps with flaporhexis, including many cases of relift for enhancement procedures with the same technique as described [2]. Our standard average flap thickness is $100 \mu \mathrm{m}$ [1] but the thinnest flap we ever lifted with flaporhexis resulted in a flap thickness of an astonishing $58 \mu \mathrm{m}$ measured by our routine online optical coherence pachymetry (subtraction method). This experience supports the theory that the major supporting structure component of the sub-Bowman's Keratomileusis (SBK) flap is Bowman's membrane. It also emphasizes the safety of this procedure as well as pointing out the biophysical robustness of thin flaps created by femtosecond technology.

As with any procedure, complications may arise. We have not, however, had a single complication that led to best correction vision accuity (BCVA) loss due to flap malformation. One critical situation that may occur is if a pronounced line of bridging tissue occurs due to eye movement while the photo disruption process (Figure 13) of the femto laser is in progress. When tearing on the flap, the surgeon will feel the slight resistance of the tissue bridge that is overcome by the momentum of the tearing. A surgeon very rarely needs to abandon flaporhexis: In the case of resistance to flap lifting being too high, the surgeon is advised to start carefully. We have experienced

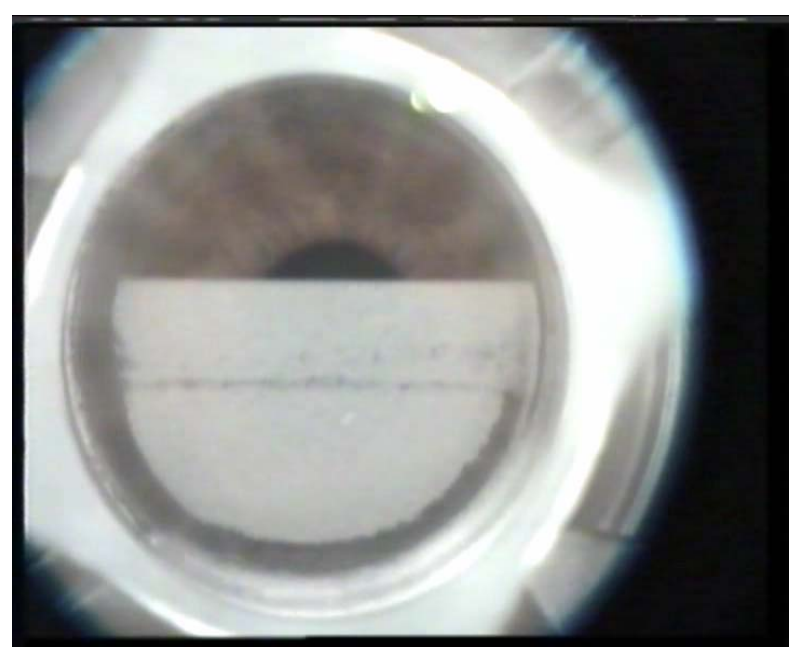

Figure 13. Line of tissue bridging during the photo disruption process of the femtosecond laser. 
a total of 5 incidents of tissue bite-out (Figure 7) or flap tears (Figures 8 and 9) caused by incomplete side cuts, the use of the wrong or too-sharp forceps or the euphoria of the surgeon getting taken away with the new technique. However, they were repaired by inserting the flap particle after refloating the flap, just like an inlay in a veneer. A therapeutic contact lens should be placed for safety considerations the first night in this case just as Pallikaris recommended in his historic description of LASIK [6]. We would like to stress this possibility of complications because the authors of the article mentioned earlier [3] have obviously not yet realized the impending danger of a tear in a flap that had never experienced a complete delamination like in femtosecond laser flap making. Sometimes a combination of both techniques is necessary. In the case of an incomplete side cut, many surgeons are prone to give up the operation, postpone it or convert it to PRK [3]. Many such occurrences can happen to any experienced surgeon. Of course one can go back to the femtolaser, insert the docking device, decrease the flap size and restart the procedure or do a "side cut only" procedure, but always with the impending risk of creating "onion rings" when the flap is to be lifted after the femtolaser.

We suggest the following alternative, and have safely proceeded with a combination of hinge opening and flaporhexis, because here you can experience the flaporhexis at a glance. Start the separation of the flap in the hinge-opening technique till the entire flap, but only the incomplete side cut, is delaminated. We have seen colleagues stuck in this situation taking a pair of corneal scissors to cut open the missing open edge by hand. Alternatively, it is suggested to grab the flap not at the 6 o'clock position but at the end of the incomplete side cut. Now again, just as in capulorhexis [5], take control of the tension vector and pull the flap back to the hinge, release and regrab it at each successive two-hour position in order not to lose control over the rhexis.

In the beginning we faced problems performing an absolutely safe flaporhexis by using all sorts of sharp edged forceps designs available in our different operation sets. Those interested in flaporhexis should have a close look at the design of the flaporhexis forceps (Figure 1). At first glance it appears bulky, but there are no sharp edges. The forceps properly applied take away all stress from the flap's edge, but disperse the tension to the center of the cornea and from there towards the hinge.

If problems arise when performing flaporhexis, the surgeon can stop at any point of the procedure and switch back to the technique of hinge opening. It is therefore an easy technique to learn - even for beginners. In the earlier stages of femtosecond flap creation (e.g. with the 5, 10 , and $15 \mathrm{~Hz}$ technologies), when photo disruption was not as smooth and perfect as today surgeons were taught and many still use the hinge opening technique. It is still a safe access to flap creation although the mechanical tissue stress is evident when a surgeon is converting from mechanical microkeratomes to the "bladeless" technique. Thanks to the new, advanced femtosecond lasers, the improved quality of tissue dissection flaporhexis is fast and effective. We believe that this technique is especially appealing to any surgeon who combines wavefront-guided treatments in combination with femtosecond laser technology.

\section{Summary}

Very recently, an article [3] pinpointed the secrets of flaporhexis as "rapid and effective". Flaporhexis has now reached a higher level of publicity. Fast and more precise femtosecond laser technology is available and as more colleagues get interested in the flaporhexis technique, it will help to make "primary" femto LASIK and retreatment cases more rapid and effective with fewer side effects.

\section{REFERENCES}

[1] W. A. Pfaeffl, M. Kunze, U. Zenk, M. B Pfaeffl, T. Schuster and C. Lohmann, "Predictive Factors of Femtosecond Laser Flap Thickness Measured by Online Optical Coherence Pachymetry Subtraction in Sub-Bowman Keratomileusis," Journal of Cataract \& Refractive Surgery, Vol. 34, No. 1, 2008, pp. 1872-1880. doi:10.1016/j.jcrs.2008.07.017

[2] J. L. Febbraro, K. A. Buzard and M. H. Friedlander, "Reoperations after Myopic Laser in Situ Keratomileusis," Journal of Cataract \& Refractive Surgery, Vol. 26, No. 1, 2000, pp. 41-48. doi:10.1016/S0886-3350(99)00263-1

[3] S. E. Wilson and M. R. Santhiago, "Flaporhexis: Rapid and Effective Technique to Limit Epithelial Ingrowth after LASIK Enhancement," Journal of Cataract \& Refractive Surgery, Vol. 38, No. 1, 2012, pp. 2-4. doi:10.1016/j.jcrs.2011.10.015

[4] P. S. Binder, "One Thousand Consecutive IntraLase Laser in Situ Keratomileusis Flaps," Journal of Cataract \& Refractive Surgery, Vol. 32, No. 6, 2006, pp. 962-969. doi:10.1016/j.jcrs.2006.02.043

[5] H. V. Gimbel and T. Neuhann, "Development, Advantages, and Methods of the Continuous Circular Capsulorhexis Technique," Journal of Cataract \& Refractive Surgery, Vol. 16, No. 1, 1990, pp. 31-37.

[6] I. G. Pallikaris, M. E. Papatzanaki, E. Z. Stathi, O. Frenschock and A. Georgiadis, "Laser in Situ Keratomileusis," Lasers in Surgery and Medicine, Vol. 10, No. 5, 1990, pp. 463-468. doi:10.1002/1sm.1900100511 